\title{
Systemic Anticoagulation is Associated With Decreased Mortality in COVID-19 Patients: A Propensity Score-Matched Cohort Study
}

\section{Yi Bian}

Department of Critical Care Medicine, Tongji Hospital of Tongji Medical College of Huazhong University of Science and Technology

\section{Yue Le}

Department of Critical Care Medicine, Tongji Hospital of Tongji Medical College of Huazhong University of Science and Technology

\section{Ping Zhang}

Department of Neurology, Tongji Hospital of Tongji Medical College of Huazhong University of Science and Technology

\section{Zhigang He}

Department of Critical Care Medicine, Tongji Hospital of Tongji Medical College of Huazhong University of Science and Technology

\section{Ye Wang}

Department of Critical Care Medicine, Tongji Hospital of Tongji Medical College of Huazhong University of Science and Technology

\section{Shanshan Yu}

Department of Critical Care Medicine, Tongji Hospital of Tongji Medical College of Huazhong University of Science and Technology

\section{Yu Fang}

Department of Critical Care Medicine, Tongji Hospital of Tongji Medical College of Huazhong University of Science and Technology

\section{Gang Yu}

Tongji Hospital of Tongji Medical College of Huazhong University of Science and Technology

\section{Jianmin Ling}

Department of Critical Care Medicine, Tongji Hospital of Tongji Medical College of Huazhong University of Science and Technology

\section{Yikuan Feng}

Department of Critical Care Medicine, Tongji Hospital of Tongji Medical College of Huazhong University of Science and Technology

\section{Sheng Wei}


Department of Epidemiology and Biostatistics, Ministry of Education Key Laboratory of Environment and Health,School of Public Health, Tongji Medical College, Huazhong University of Science and Technology

\section{Jiao Huang}

Center for Evidence-Based and Translation Medicine, Zhongnan Hospital of Wuhan

\section{Liuniu Xiao}

Department of Critical Care Medicine, Tongji Hospital of Tongji Medical College of Huazhong University of Science and Technology

\section{Yingfang Zheng}

Department of Critical Care Medicine, Tongji Hospital of Tongji Medical College of Huazhong University of Science and Technology

\section{Zhen Yu}

Department of Critical Care Medcine, Tongji Hospital of Tongji Medical College of Huazhong University of Science and Technology

\section{Shusheng Li ( $\nabla$ lishusheng@hust.edu.cn )}

Department of Critical Care Medicine, Tongji Hospital, Tongji Medical College, Huazhong University of Science and Technology https://orcid.org/0000-0002-8799-6394

\section{Research Article}

Keywords: Coronavirus, COVID-19, anticoagulation, heparin, coagulopathy, mortality

Posted Date: July 10th, 2021

DOl: https://doi.org/10.21203/rs.3.rs-688472/v1

License: (9) This work is licensed under a Creative Commons Attribution 4.0 International License. Read Full License 


\section{Abstract}

Background: Accumulating evidence has revealed that coagulopathy and widespread thrombosis in the lung are common in patients with Coronavirus Disease 2019 (COVID-19). This raises questions about the efficacy and safety of systemic anticoagulation (AC) in COVID-19 patients.

Method: This single-center, retrospective, cohort study unselectively reviewed 2272 patients with COVID19 admitted to the Tongji Hospital between Jan 25 and Mar 23, 2020. Propensity score-matching between patients adjusted for potential covariates was carried out with the patients divided into two groups depending on whether or not they had received AC treatment (AC group, ${ }^{37}$ days of treatment; nonAC group, no treatment). This yielded 164 patients in each group.

Result: In-hospital mortality of the AC group was significantly lower than that of the non-AC group (14.0\% vs. $28.7 \%, P=0.001)$. Treatment with $\mathrm{AC}$ was associated with a significantly lower probability of inhospital death (adjusted HR=0.273, $95 \% \mathrm{Cl}, 0.154$ to $0.484, P<0.001$ ). The incidence of major bleeding and thrombocytopenia in the two groups was not significantly different. Subgroup analysis showed the following factors were associated with a significantly lower in-hospital mortality in patients who had received $A C$ treatment; severe cases ( $13.2 \%$ vs. $24.6 \%, P=0.018)$, critical cases $(20.0 \%$ vs $82.4 \%, P=0.003)$, patients with a D-dimer level $\geq 0.5 \mu \mathrm{g} / \mathrm{mL}$ ( $14.8 \%$ vs. $33.8, P<0.001)$, and moderate $(16.7 \%$ vs. $60.0 \%$, $P=0.003$ ) or severe acute respiratory distress syndrome (ARDS) cases at admission (33.3\% vs. $86.7 \%$, $P=0.004)$. During the hospital stay, critical cases $(38.3 \%$ vs. $76.7 \%, P<0.001)$ and severe ARDS cases $(36.5 \%$ vs. $76.3 \%, P<0.001)$ who received AC treatment had significantly lower in-hospital mortality.

Conclusions: AC treatment decreases the risk of in-hospital mortality, especially in critically ill patients, with no additional significant, major bleeding events or thrombocytopenia being observed.

\section{Trials registration - ChiCTR2000039855}

\section{Introduction}

Coronavirus disease 2019 (COVID-19) has developed into a pandemic disease and affected nearly every country in the world. There is no comprehensive and strong clinical evidence to support the efficacy of any drugs that specifically target the severe acute respiratory syndrome coronavirus 2 (SARS-CoV-2)[1]. Previous research has found that coagulopathy is very common in COVID-19 patients, and includes thrombosis and coagulation abnormalities and dysfunction such as an elevated D-dimer level and prolonged prothrombin time (PT), respectively[2]. Autopsy histopathologic analysis has identified widespread thrombosis and microangiopathy in small vessels and capillaries of the lung $[3 ; 4 ; 5]$, that are different from the pathologies observed in respiratory failure caused by other diseases $[3 ; 6 ; 7 ; 8]$. Some scholars have therefore proposed anticoagulation (AC) treatment as an integral part of systemic therapy in the early stage of COVID-19[9]. Retrospective research has suggested that AC may decrease mortality in COVID-19 patients[9; 10], although these conclusions are not completely reliable nor applicable to all COVID-19 patients due to limitations in methodology such as no prospective control or matching cohort, 
large heterogeneity in anticoagulant therapy, and a lack of subgroup analysis. As a consequence, the recommendations for empiric systemic AC treatment currently differ between COVID-19 management guidelines $[11 ; 12 ; 13]$, with some recommending to use anticoagulant drugs preventively for patients with no contraindications to AC and a significantly increased D-dimer level, while others recommend that all hospitalized adults with COVID-19 should receive pharmacologic thromboprophylaxis with low molecular weight heparin (LMWH) rather than unfractionated heparin (UFH).

We conducted a propensity score-matched cohort study using a comprehensive database of COVID-19 patients to investigate whether AC treatment was protective and safe for COVID-19 patients. Innovative analyses were carried out in which the propensity score (PS) matching was performed by balancing demographic variates, disease severity, and major treatment between patients with or without AC treatment. A second aim of the study was to identify the patients that benefited most from AC treatment using subgroup analysis that involved stratifying the data according to the severity of the acute respiratory distress syndrome (ARDS)[14], COVID-19 clinical classification[13], and D-dimer levels.

\section{Materials And Methods}

\subsection{Ethics and registration}

This retrospective cohort study was approved by the ethics committee of Tongji Medical College, Huazhong University of Science and Technology (No. 2020-S220). The clinical trial was registered and verified by the Chinese Clinical Trial Registry (ChiCTR2000039855).

\subsection{Patient population and study design}

This single-center retrospective cohort study was conducted in two exclusive branches of COVID-19 patients treated at Tongji Hospital, an academic hospital affiliated to Tongji Medical College, Huazhong University of Science and Technology in Wuhan, China. All patients with confirmed COVID-19 admitted consecutively to these two institutions between Jan 25 to Mar 23, 2020 were enrolled retrospectively in the study. Approval was obtained from the ethics committee at our institution that the patients did not have to provide informed consent for inclusion in the study. Patients who received systemic therapeutic dose $A C$ treatment for at least 7 days during hospitalization were assigned to the $A C$ group, while patients who did not receive any AC treatment were assigned to the non-AC group. The medications administered and clinical outcomes were followed-up to June 4, 2020, when these two branches for exclusive COVID19 treatment were closed. All COVID-19 patients were diagnosed according to the World Health Organization interim guidelines[15] and the Diagnosis and Treatment Protocol for COVID-19 Patients (Trial Version 8)[13]. The exclusion criteria for the study were younger than 18 years, pregnant, length of stay $<24$ hours, insufficient medical information, a history of severe comorbidities requiring surgical operation including, but not limited to, multiple trauma, a severe infection that required debridement, amputation or laparotomy, and patients who were classified again as COVID positive after RNA for SARSCoV-2 was detected following their discharge from hospital. 
To minimize bias caused by the nonrandom allocation of potential confounding covariates between the AC and non-AC groups, we adopted PS matching[16] methods. PS was calculated using a logistic regression model, adjusted for the following covariates: level of oxygen therapy, clinical classification, high-sensitivity C reactive protein (hs-CRP) and D-dimer levels, CURB-65 score for the severity of pneumonia[17] at hospital admission, and the highest level of oxygen therapy during hospitalization. The match ratio was set at 1 to 1 and the maximum allowable distance (caliper) at 0.1[18].

\subsection{Outcomes, definitions and data collection}

AC treatment was defined as receiving either therapeutic-dose UFH (intravenous), LMWH (subcutaneous injection) or direct-acting oral anticoagulants (DOACs) (mainly Rivaroxaban and Argatroban) for at least 7 days.

The primary outcome of this study was in-hospital mortality. The safety endpoints included bleeding events and thrombocytopenia. Bleeding events included major bleeding, gastrointestinal bleeding, hemoptysis, hematuria, and bleeding in other parts. Major bleeding was defined according to the International Society on Thrombosis and Haemostasis statement[19] as those that resulted in death, were life-threatening, caused chronic sequelae or consume major healthcare resources. All other bleeding episodes were classified as minor bleeding[20]. Thrombocytopenia was defined as a platelet count $<100 \times 10^{9} / L[21]$.

The CURB-65 score[17; 22], ARDS[14; 23; 24] and quick sequential organ failure assessment (qSOFA) [25] were defined according to the literature, while the COVID-19 clinical classification was made according to the Diagnosis and Treatment Protocol for COVID-19 Patients (Trial Version 8)[13].

All the characteristics and clinical information of the patients were obtained from electronic medical and nursing record systems. This data included age, gender, current smoking history, comorbidities, laboratory results at admission, CURB-65 score and qSOFA score at admission, ARDS classification and COVID-19 clinical classification at admission and during the hospital stay, antiviral therapies and other treatments during hospitalization, the level of oxygen therapy at admission, and the most intense level of oxygen therapy during hospitalization.

\subsection{Statistical analysis}

Quantitative variables were expressed as medians and interquartile ranges (IQR) and compared using the Mann-Whitney $U$ test. Categorical variables were compared using the Pearson $\chi 2$ test, continuity correction, or Fisher's exact test, as appropriate. A Kaplan-Meier curve was used to analyse survival during hospitalization, with the data stratified according to AC treatment. Univariate Cox proportional hazards regression was used to determine the risk factors for in-hospital mortality. Factors with a $P$ value $<0.05$ were then included in a multivariate Cox proportional hazard regression model. SPSS version 22.0 software (IBM Corp., Armonk, New York, U.S.) was used for the statistical analyses and PS matching. The Kaplan-Meier survival plot was constructed using GraphPad Prism version 4.0 software (GraphPad 
Software Inc., La Jolla, CA, USA). All tests were two-tailed, with a $P$-value $<0.05$ considered statistically significant.

\section{Results}

\subsection{Clinical characteristics of the patients at presentation}

2469 confirmed COVID-19 patients were admitted to Tongji Hospital between Jan 25 and Mar 23, 2020. Exclusion of 197 patients who did met the study entry criteria left 2272 patients to be consecutively and unselectively identified as candidates for PS matching. In this cohort, 78 patients who received AC treatment for $<7$ days were excluded before PS matching. Finally, PS matching yielded 164 patients in the $\mathrm{AC}$ group and 164 in the non-AC group after adjusting for covariates between the two groups (Figure 1). Compared to the non-AC group, patients in the AC group were older (69 [60-78] yr vs 67 [56-73] yr, $P=0.017)$ and had more comorbidities at admission ( $76.2 \%$ vs $61.6 \%, P=0.004)$, higher white blood cell counts $\left(7.05 \times 10^{9} / \mathrm{L}\right.$ vs $\left.6.52 \times 10^{9} / \mathrm{L} \otimes P=0.044\right)$, higher neutrophil counts $(5.56 \times 109 / \mathrm{L}$ vs $4.84 \times 109 / \mathrm{L}$, $P=0.035)$, and higher $D$-dimer levels $(2.26 \mu \mathrm{g} / \mathrm{mL}$ vs $1.40 \mu \mathrm{g} / \mathrm{mL}, P=0.003)$. At hospital admission, no significant difference in oxygen therapy, COVID-19 clinical classification, and ARDS classification was observed between the two groups. As shown in Table 1, a higher proportion patients in the AC group were receiving intravenous immunoglobulin (IVIG) $(50.0 \%$ vs $31.1 \%, P<0.001)$, corticosteroid $(64.0 \%$ vs $51.8 \%$, $P=0.025)$, and convalescent plasma ( $8.5 \%$ vs $1.8 \%, P=0.006)$ than patients in the non-AC group. The median duration of hospitalization in all the patients was 28 days (IQR, 15-41days), while the median duration of $A C$ treatment was 15 days (IQR, 10-22 days).

Variables represented the poorest value of the first day at admission. $A C=$ anticoagulation; IQR=interquartile range; $A R D S=$ acute respiratory distress syndrome; qSOFA=quick sequential organ failure assessment; hs-CRP=high sensitive $\mathrm{C}$ reacting protein; $\mathrm{IVIG=intravenous} \mathrm{immunoglobulin;}$ $\mathrm{ECMO}=$ extracorporeal membrane oxygenation

\subsection{Primary and secondary outcomes}

In-hospital mortality in the AC group was significantly lower than that of the non-AC group (23/164 [14.0\%] vs. 47/164 [28.7\%], $P=0.001)$. Secondary outcomes included bleeding events and thrombocytopenia. Patients receiving AC treatment had a higher rate of total bleeding events than patients in the non-AC group ( $25.0 \%$ vs. $11.0 \%, P=0.001)$, especially for hemoptysis $(6.1 \%$ vs. $1.2 \%$, $P=0.019)$ and hematuria ( $18.3 \%$ vs. $8.5 \%, P=0.010)$. However, there was no significant difference in major bleeding events ( 0 vs. $1.2 \%, P=0.498$ ) and thrombocytopenia ( $15.2 \%$ vs. $14.6 \%, P=0.755)$ between the two groups (Table 2). 
Table 1. Patients Baseline Characteristics and Treatments

No. $(\%)$

$\begin{array}{llll}\begin{array}{l}\text { Total } \\ (\mathrm{n}=328)\end{array} & \begin{array}{c}\text { AC treatment } \\ (\mathrm{n}=164)\end{array} & \begin{array}{l}\text { Non-AC } \\ \text { treatment } \\ (\mathrm{n}=164)\end{array} & P \\ & & & \text { value }\end{array}$

Age, median (IQR), years

$68(58-75) \quad 69(60-78)$

$67(56-73)$

0.017

Gender

Male

186 (56.7) $84(51.2)$

$102(62.2)$

0.045

Female

$142(43.3) \quad 80(48.8)$

$62(37.8)$

Current smoking

$31(9.5)$

19 (11.6)

$12(7.3)$

0.186

Comorbidities

226 (68.9) 125 (76.2)

101 (61.6)

0.004

Diabetes

$70(21.3) \quad 36(22.0)$

34 (20.7)

0.788

Hypertension

$161(49.1) \quad 93(56.7)$

68 (41.5)

0.006

Cardiovascular disease

$48(14.6) \quad 32(19.5)$

$16(9.8)$

0.012

Chronic obstructive pulmonary

$34(10.4) \quad 18(11.0)$

$16(9.8)$

0.717

disease

Chronic kidney disease

$11(3.4)$

$8(4.9)$

$3(1.8)$

0.125

Chronic liver disease

$16(4.9)$

$5(3.0)$

$11(6.7)$

0.124

Autoimmune disease

4 (1.2)

$2(1.2)$

2 (1.2)

1.000

Immunosuppression

$1(0.3)$

$0(0)$

$1(0.6)$

1.000

Malignancy

$12(3.7)$

$3(1.8)$

9 (5.5)

0.078

Oxygen therapy at admission

Without oxygen inhalation

$56(17.1) \quad 25(15.2)$

$31(18.9)$

0.462

Nasal cannula

$213(64.9) \quad 107$ (65.2)

$106(64.6)$

Face mask with reservoir bag

$33(10.1)$

$20(12.2)$

$13(7.9)$

High-flow nasal cannula

$3(0.9)$

$2(1.2)$

$1(0.6)$

Noninvasive ventilation(bi-level)

$15(4.6)$

5 (3.0)

$10(6.1)$

Invasive mechanical ventilation

$8(2.4)$

$5(3.0)$

$3(1.8)$

ARDS at admission

No ARDS

Mild
$173(52.7) \quad 82(50.0)$

$84(25.6) \quad 46(28.0)$

Page $7 / 23$ 


\begin{tabular}{llll} 
Moderate & $44(13.4)$ & $24(14.6)$ & $20(12.2)$ \\
\hline Severe & $27(8.2)$ & $12(7.3)$ & $15(9.1)$
\end{tabular}

Clinical classification at admission

\begin{tabular}{lllll}
\hline Moderate & $35(10.7)$ & $18(11.0)$ & $17(10.4)$ & \multirow{2}{*}{0.372} \\
\cline { 1 - 3 } Severe & $266(81.1)$ & $136(82.9)$ & $130(79.3)$ & \\
\cline { 1 - 3 } Critical & $27(8.2)$ & $10(6.1)$ & $17(10.4)$ & \\
\hline CURB-65 score at admission & $1(0-2)$ & $1(0-2)$ & $1(0-2)$ & 0.593 \\
\hline qSOFA score at admission & $0(0-1)$ & $0(0-1)$ & $0(0-1)$ & 0.215 \\
\hline
\end{tabular}

Initial laboratory parameters,

median (IQR)

\begin{tabular}{|c|c|c|c|c|}
\hline White blood cells, $\times 10^{9} / \mathrm{L}$ & $\begin{array}{l}6.77(5.09- \\
9.04)\end{array}$ & $7.05(5.39-9.48)$ & $6.52(4.78-8.59)$ & 0.044 \\
\hline Neutrophils, $\times 10^{9} / \mathrm{L}$ & $\begin{array}{l}5.35(3.52- \\
7.59)\end{array}$ & $5.56(3.99-7.68)$ & $4.84(3.04-716)$ & 0.035 \\
\hline Lymphocytes, $\times 10^{9} / \mathrm{L}$ & $\begin{array}{l}0.81(0.56- \\
1.17)\end{array}$ & $0.84(0.58-1.16)$ & $0.78(0.5-1.17)$ & 0.239 \\
\hline Platelets, $\times 10^{9} / \mathrm{L}$ & $\begin{array}{l}200.5 \\
(145.0- \\
270.8)\end{array}$ & $\begin{array}{l}209.5 \text { (145.3- } \\
278.5)\end{array}$ & $\begin{array}{l}193.5(145.0- \\
269.8)\end{array}$ & 0.477 \\
\hline Total bilirubin, mmol/L & $\begin{array}{l}9.7(7.2- \\
14.0)\end{array}$ & $10.4(7.0-14.0)$ & $9.4(7.4-14.0)$ & 0.681 \\
\hline Lactate dehydrogenase, U/L & $\begin{array}{l}339(252- \\
473)\end{array}$ & $341(261-465)$ & $339(246-480)$ & 0.460 \\
\hline Urea, mmol/L & $\begin{array}{l}5.10(3.90- \\
7.90)\end{array}$ & $5.20(4.00-7.75)$ & $5.00(3.80-8.00)$ & 0.721 \\
\hline hs-CRP, mg/L & $\begin{array}{l}51.7(17.0- \\
110.6)\end{array}$ & $14.1(13.5-15.2)$ & $14.0(13.4-15.1)$ & 0.657 \\
\hline Prothrombin time, s & $\begin{array}{l}14.1(13.4- \\
15.1)\end{array}$ & $53.3(17.0-109.5)$ & $51.7(15.6-113.0)$ & 0.542 \\
\hline D-dimer, mg/mL & $\begin{array}{l}1.79(0.81- \\
4.49)\end{array}$ & $2.26(1.10-5.31)$ & $1.40(0.59-3.78)$ & 0.003 \\
\hline \multicolumn{5}{|l|}{ Antiviral therapy } \\
\hline Interferon & $32(9.8)$ & $11(6.7)$ & $21(12.8)$ & 0.063 \\
\hline Oseltamivir & $98(29.9)$ & $48(29.3)$ & $50(30.5)$ & 0.809 \\
\hline
\end{tabular}




\begin{tabular}{|c|c|c|c|c|}
\hline Arbidol & $262(79.9)$ & $132(80.5)$ & $130(79.3)$ & 0.783 \\
\hline Lopinavir & $83(25.3)$ & $44(26.8)$ & $39(23.8)$ & 0.525 \\
\hline Ribavirin & $47(14.3)$ & $25(15.2)$ & $22(13.4)$ & 0.636 \\
\hline Hydroxychloroquine & $47(14.3)$ & $28(17.1)$ & 19 (11.6) & 0.156 \\
\hline Traditional Chinese medicine & $254(77.4)$ & $134(81.7)$ & $120(73.2)$ & 0.064 \\
\hline \multicolumn{5}{|l|}{ Other treatments } \\
\hline IVIG & $133(40.5)$ & $82(50.0)$ & $51(31.1)$ & $<0.001$ \\
\hline Corticosteroid & $190(57.9)$ & $105(64.0)$ & $85(51.8)$ & 0.025 \\
\hline Convalescent plasma & $17(5.2)$ & $14(8.5)$ & $3(1.8)$ & 0.006 \\
\hline \multicolumn{5}{|l|}{$\begin{array}{l}\text { The most intense oxygen therapy } \\
\text { during hospitalization }\end{array}$} \\
\hline Without oxygen inhalation & $4(1.2)$ & $1(0.6)$ & $3(1.8)$ & \multirow[t]{7}{*}{0.002} \\
\hline Nasal cannula & $170(51.8)$ & $85(51.8)$ & $85(51.8)$ & \\
\hline Face mask with reservoir bag & $22(6.7)$ & $15(9.1)$ & $7(4.3)$ & \\
\hline High-flow nasal cannula & $16(4.9)$ & $7(4.3)$ & $9(5.5)$ & \\
\hline Noninvasive ventilation(bi-level) & $46(14.0)$ & $13(7.9)$ & $33(20.1)$ & \\
\hline Invasive mechanical ventilation & $63(19.2)$ & $36(22.0)$ & $27(16.5)$ & \\
\hline ECMO & $7(2.1)$ & $7(4.3)$ & $0(0)$ & \\
\hline
\end{tabular}


Table 2. Primary and secondary outcomes

No. $(\%)$

Total $(n=328) \quad$ AC group $(n=164) \quad$ non-AC group $(n=164) \quad P$ value

Primary outcomes

In-hospital mortality

$70(21.3)$

$23(14.0)$

$47(28.7)$

0.001

Secondary outcomes

\begin{tabular}{lllll}
\hline Bleeding events & $59(18.08)$ & $41(25.0)$ & $18(11.0)$ & 0.001 \\
\hline Major bleeding & $2(0.6)$ & $0(0)$ & $2(1.2)$ & 0.498 \\
\hline Minor bleeding & & & $3(1.8)$ & 0.078 \\
\hline Gastrointestinal bleeding & $12(3.7)$ & $9(5.5)$ & $2(1.2)$ & 0.019 \\
\hline Hemoptysis & $12(3.7)$ & $10(6.1)$ & $14(8.5)$ & 0.010 \\
\hline Hematuria & $44(13.4)$ & $30(18.3)$ & $0(0)$ & 0.131 \\
\hline Bleeding in other parts & $4(1.2)$ & $4(2.4)$ & $24(14.6)$ & 0.755
\end{tabular}

The median survival time was longer in the AC group compared to that in the non-AC group ( 36 days, IQR $23-45$ vs. 22 days, IQR 10-31, $P<0.001)$. Univariate Cox proportional hazard regression analysis showed a significantly lower probability of in-hospital death in the AC treatment group compared to the non-AC treatment group (hazard ratio $[\mathrm{HR}]=0.362 ; 95 \% \mathrm{Cl}, 0.219$ to $0.598 ; P<0.001$ ). The univariate Cox analysis showed that the following factors at admission were risk factors for in-hospital mortality; greater age ( $\mathrm{HR}=1.033,95 \% \mathrm{Cl} 1.012$ to $1.054, P=0.002)$, use of IVIG (HR=1.610, $95 \% \mathrm{Cl} 1.003$ to $2.587, P=0.049)$, use of corticosteroid ( $\mathrm{HR}=2.915,95 \% \mathrm{Cl} 1.593$ to $5.332, \mathrm{P}=0.001)$, elevated total bilirubin $(\mathrm{HR}=1.019,95 \% \mathrm{Cl}$ 1.001 to $1.038, P=0.040$ ), elevated lactate dehydrogenase ( $H R=1.003,95 \% \mathrm{Cl} 1.002$ to $1.003, P<0.001)$, elevated urea $(\mathrm{HR}=1.096,95 \% \mathrm{Cl} 1.068$ to $1.125, P<0.001)$, elevated prothrombin time $(\mathrm{HR}=1.088,95 \% \mathrm{Cl}$ 1.049 to $1.128, P<0.001$ ), elevated hs-CRP ( $\mathrm{HR}=1.008,95 \% \mathrm{Cl} 1.005$ to $1.011, P<0.001$ ), and elevated $\mathrm{D}-$ dimer (HR=1.069, 95\% Cl: 1.040 to 1.098, $P<0.001)$. This analysis also showed that the use of Arbidol $(\mathrm{HR}=0.363,95 \% \mathrm{Cl} 0.223$ to $0.590, P<0.001)$ and traditional Chinese medicine ( $\mathrm{HR}=0.257,95 \% \mathrm{Cl} 0.161$ to $0.412, P<0.001)$ and elevated platelet count $(\mathrm{HR}=0.991,95 \% \mathrm{Cl} 0.988$ to $0.995, P<0.001)$ were protective factors, while the presence of comorbidities ( $\mathrm{HR}=1.059,95 \% \mathrm{Cl} 0.630$ to $1.780, P=0.830$ ) was not a risk factor. Extracorporeal membrane oxygenation ( $\mathrm{HR}=0.857,95 \% \mathrm{Cl} 0.207$ to $3.553, P=0.832)$ and convalescent plasma application ( $\mathrm{HR}=0.416,95 \% \mathrm{Cl} 0.102$ to $1.701, P=0.222)$ were shown not to protective factors for in-hospital mortality.

As shown in figure 2, multivariate Cox analysis revealed AC treatment was associated with a lower probability of in-hospital mortality (adjusted HR=0.273, $95 \% \mathrm{Cl} 0.154$ to $0.484, P<0.001$ ), after adjusting 
for variables including age, the use of either Arbidol, traditional Chinese medicine, IVIG, or corticosteroid, platelets count, and the levels of PT, D-dimer, total bilirubin, lactate dehydrogenase, urea, and hs-CRP. In this multivariate model, elevated platelet count (adjusted HR=0.996, $95 \% \mathrm{Cl} 0.993$ to $0.999, P=0.016$ ), and the use of Arbidol (adjusted HR=0.491, 95\% Cl 0.284 to $0.851 P=0.011$ ) or traditional Chinese medicine (adjusted HR=0.409, 95\% Cl: 0.239 to $0.700, P=0.001$ ) remained protective factors. Furthermore, IVIG (adjusted $\mathrm{HR}=1.794,95 \% \mathrm{Cl} 1.060$ to $3.035, P=0.029$ ) and use of corticosteroid (adjusted HR=2.097, 95\% $\mathrm{Cl} 1.102$ to $3.992, P=0.024$ ) remained independent risk factors for in-hospital death.

\subsection{Subgroup stratification}

In-hospital mortality between the AC treatment and the non-AC treatment groups was compared in individuals stratified according to ARDS classification, COVID-19 clinical classification, and D-dimer levels at both hospital admission and during hospitalization (Table 3 ). 
Table 3. The association between AC treatment and in-hospital mortality in stratified patients

In hospital mortality (\%)

\begin{tabular}{|c|c|c|c|c|c|}
\hline \multirow{2}{*}{$\begin{array}{l}\text { Total } \\
(\mathrm{n}=328)\end{array}$} & \multirow{2}{*}{$\begin{array}{l}\text { AC group } \\
(n=164)\end{array}$} & \multirow{2}{*}{$\begin{array}{l}\text { Non-AC } \\
\text { group } \\
(n=164)\end{array}$} & \multirow{2}{*}{$\begin{array}{l}P \\
\text { value }\end{array}$} & \multirow[t]{2}{*}{ OR } & $95 \% \mathrm{Cl}$ \\
\hline & & & & & $\begin{array}{l}\text { Lower } \\
\text { Bound }\end{array}$ \\
\hline
\end{tabular}

At admission

ARDS

classification

$\begin{array}{llllllll}\text { No ARDS } & 14 / 173 & 5 / 82(6.1) & 9 / 91(9.9) & 0.361 & 0.592 & 0.190 & 1.844\end{array}$

$(8.1)$

$\begin{array}{llllllll}\text { Mild } & 23 / 84 & 10 / 46 & 13 / 38(34.2) & 0.202 & 0.534 & 0.203 & 1.409\end{array}$

(27.4) (21.7)

Moderate $\quad 16 / 44 \quad 4 / 24$

(36.4) (16.7)

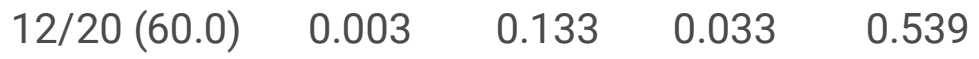

Severe

$17 / 27 \quad 4 / 12$

(63.0) (33.3)

$\begin{array}{lllll}13 / 15(86.7) & 0.004 & 0.077 & 0.011 & 0.521\end{array}$

COVID-19 Clinical

classification

\begin{tabular}{|c|c|c|c|c|c|c|c|}
\hline Moderate & $\begin{array}{l}4 / 35 \\
(11.4)\end{array}$ & $\begin{array}{l}3 / 18 \\
(16.7)\end{array}$ & $1 / 17(5.9)$ & 0.603 & 3.200 & 0.299 & 34.241 \\
\hline Severe & $\begin{array}{l}50 / 266 \\
(18.8)\end{array}$ & $\begin{array}{l}18 / 136 \\
(13.2)\end{array}$ & $\begin{array}{l}32 / 130 \\
(24.6)\end{array}$ & 0.018 & 0.467 & 0.247 & 0.883 \\
\hline Critical & $\begin{array}{l}16 / 27 \\
(59.3)\end{array}$ & $\begin{array}{l}2 / 10 \\
(20.0)\end{array}$ & $14 / 17(82.4)$ & 0.003 & 0.054 & 0.007 & 0.391 \\
\hline
\end{tabular}

D-dimer, $\mu \mathrm{g} / \mathrm{mL}$

\begin{tabular}{|c|c|c|c|c|c|c|c|}
\hline D-dimer $<0.5$ & $\begin{array}{l}1 / 37 \\
(2.7)\end{array}$ & $0 / 9(0)$ & 1/28 (3.6) & 1.000 & 0.750 & 0.621 & 0.906 \\
\hline D-dimer $\geq 0.5$ & $\begin{array}{l}69 / 291 \\
(23.7)\end{array}$ & $\begin{array}{l}23 / 155 \\
(14.8)\end{array}$ & $\begin{array}{l}46 / 136 \\
(33.8)\end{array}$ & $<0.001$ & 0.341 & 0.193 & 0.601 \\
\hline $0.5 \leq$ D-dimer $<2.5$ & $\begin{array}{l}29 / 159 \\
(18.2)\end{array}$ & $\begin{array}{l}8 / 78 \\
(10.3)\end{array}$ & $21 / 81(25.9)$ & 0.011 & 0.327 & 0.135 & 0.791 \\
\hline $2.5 \leq \mathrm{D}$-dimer $<5.0$ & $\begin{array}{l}12 / 53 \\
(22.6)\end{array}$ & $\begin{array}{l}4 / 34 \\
(11.8)\end{array}$ & $8 / 19(42.1)$ & 0.017 & 0.183 & 0.046 & 0.732 \\
\hline D-dimer $\geq 5.0$ & $\begin{array}{l}28 / 79 \\
(35.4)\end{array}$ & $\begin{array}{l}11 / 43 \\
(25.6)\end{array}$ & $17 / 36(47.2)$ & 0.045 & 0.384 & 0.149 & 0.991 \\
\hline
\end{tabular}

During

hospitalization 
ARDS

classification

\begin{tabular}{|c|c|c|c|c|c|c|c|}
\hline No ARDS & $0 / 58(0)$ & $0 / 21(0)$ & $0 / 37(0)$ & - & - & - & - \\
\hline Mild & $\begin{array}{l}0 / 103 \\
(0)\end{array}$ & $0 / 53(0)$ & $0 / 50(0)$ & - & - & - & - \\
\hline Moderate & $\begin{array}{l}2 / 45 \\
(4.4)\end{array}$ & $0 / 27(0)$ & 2/18 (11.1) & 0.155 & 0.372 & 0.252 & 0.549 \\
\hline Severe & $\begin{array}{l}68 / 122 \\
(55.7)\end{array}$ & $\begin{array}{l}23 / 63 \\
(36.5)\end{array}$ & $45 / 59(76.3)$ & $<0.001$ & 0.179 & 0.081 & 0.394 \\
\hline
\end{tabular}

COVID-19 Clinical

classification

\begin{tabular}{|c|c|c|c|c|c|c|c|}
\hline Moderate & $0 / 2(0)$ & $0 / 1(0)$ & $0 / 1(0)$ & - & - & - & - \\
\hline Severe & $\begin{array}{l}1 / 206 \\
(0.5)\end{array}$ & 0/103 (0) & 1/103 (0) & 1.000 & 0.498 & 0.434 & 0.571 \\
\hline Critical & $\begin{array}{l}69 / 120 \\
(57.5)\end{array}$ & $\begin{array}{l}23 / 60 \\
(38.3)\end{array}$ & $46 / 60(76.7)$ & $<0.001$ & 0.189 & 0.086 & 0.418 \\
\hline
\end{tabular}

$\mathrm{AC}=$ anticoagulation; $\mathrm{OR}=$ odds ratio; $\mathrm{Cl}=$ confidence interval; $\mathrm{ARDS}=$ acute respiratory distress syndrome

At hospital admission, moderate (4/24 [16.7\%] vs. $12 / 20$ [60.0\%], $P=0.003)$ and severe ARDS $(4 / 12$ [33.3\%] vs. $13 / 15$ [86.7\%], $P=0.004$ ) were associated with lower in-hospital mortality in patients on AC treatment compared to those who did not receive this treatment. In patients diagnosed as severe and critical COVID-19 cases at admission, those who received AC treatment had lower in-hospital mortality compared to patients in the non-AC group (18/136 [13.2\%] vs. $32 / 130$ [24.6\%], $P=0.018 ; 2 / 10$ [20.0\%] vs. $14 / 17$ [82.4\%], $P=0.003$, respectively). In patients with a D-dimer level $<0.5 \mu \mathrm{g} / \mathrm{mL}$ at hospital admission, there was no significant difference in in-hospital mortality between the $A C$ and the non-AC treatment groups (0/9 [0] vs. $1 / 28$ [3.6\%], $P=1.000)$. However, in patients with a $D$-dimer level $\geq 0.5 \mu \mathrm{g} / \mathrm{mL}$, those who received $A C$ treatment had a lower in-hospital mortality than patients who did not receive this treatment (23/155 [14.8] vs. 46/136 [33.8\%], $P<0.001$, respectively).

During hospital stay, patients in the AC group who developed severe ARDS (23/63 [36.5\%] vs. 45/59 [76.3\%], $P<0.001)$ or critical COVID-19 (23/60 [38.3\%] vs. 46/60 [76.7\%], $P<0.001)$ had lower in-hospital mortality compared to those who did not receive AC treatment.

\section{Discussion}

COVID-19 infections have affected patients globally. The International Society on Thrombosis and Hemostasis pointed out that COVID-19 patients develop a clinically significant coagulopathy, characterized by thrombocytopenia, mildly prolonged prothrombin time, and elevated serum D-dimer levels[21]. Recent research clearly indicates that coagulopathy is not only common in COVID-19 patients, 
but is also associated with increased mortality[9]. The potential mechanism for the development of coagulopathy in COVID-19 patients may be related to endothelial cell dysfunction[26] and hypoxiainduced thrombosis[27] following a SARS-CoV-2 infection. Because the endothelium plays an important role in regulating hemostasis, fibrinolysis, and vessel wall permeability, endothelial dysfunction in pulmonary microvessels may act as a trigger for immunothrombosis, resulting in coagulopathy. Histological analysis of pulmonary vessels in COVID-19 patients shows more widespread thrombosis with microangiopathy compared to that observed in patients with influenza[3]. Based on this preliminary evidence, AC treatment may be beneficial for COVID-19 patients by inhibiting thrombin generation and thereby reducing mortality. The International Society on Thrombosis and Hemostasis suggests that a prophylactic dose of LMWH should be considered in all patients who do not have any contraindications. Moreover, the Chinese Diagnosis and Treatment Protocol for COVID-19 Patients (Version 8.0) also suggested using $A C$ treatment in selected patients. However, these recommendations require additional clinical evidence to determine the association between AC treatment and outcome of COVID-19 patients, as well as clarify the indications, contradictions and optimal duration, dose, and time to use AC. We conducted this propensity score-matched cohort study using a comprehensive source of COVID-19 patients and showed that receiving a therapeutic-dose of AC for 7 days or longer was associated with a decrease in-hospital mortality in these patients, with no increase in the incidence of major bleeding events. A subgroup analysis was also carried out to identify patients who might obtain greater benefit from AC treatment. At hospital admission, patients who were diagnosed as severe or critical cases or those who had either moderate or severe ARDS or a D-dimer level $\geq 0.5 \mu \mathrm{g} / \mathrm{mL}$ were more likely to benefit from AC therapy. During hospitalization, patients who developed into a critical case or severe ARDS were more likely to benefit from AC therapy (Table 3).

To date, several research works have investigated systemic AC therapy in COVID-19 patients. A retrospective cohort study of 2,773 COVID-19 patients from the Mount Sinai Health System[28] suggested that systemic treatment-dose AC may provide COVID-19 patients with potential survival benefits by adjusting mechanical ventilation despite an increase in the risk of bleeding events. However, this singlecenter study did not match the AC and non-AC groups according to classification of disease severity. It is also important to note that the duration of hospitalization (median 5 days, IQR 3-8 days) and the course of AC treatment (median 3 days, IQR 2-7 days) were relatively short. The authors also suggested that a longer duration of AC treatment was associated with reduced mortality risk (adjusted HR of 0.86 per day). Within the current consensus on anticoagulant therapy for venous thromboembolism it is generally considered that patients with confirmed deep vein thrombosis or pulmonary embolism need LMWH treatment for at least five days followed by dabigatran or edoxaban[29]. Two studies have reported that AC treatment for 7 days or longer is associated with decreased mortality in COVID-19 patients[9; 30]. In our study, we also defined systemic AC treatment as receiving UFH, LMWH and/or DOACs for 7 days or longer. A retrospective study on 449 patients in Wuhan with severe COVID-19 [9] examined the relationship between heparin treatment for 7 days or longer and 28-day mortality. Although no difference was observed between the AC and the non-AC groups patients who meet the sepsis-induced coagulopathy (SIC) criteria or had a markedly elevated D-dimer level appeared to benefit from AC. Similar 
to the study from the Mount Sinai Health System, this study also did not match patients with or without AC treatment according to the classification of disease severity, which may have led to unbalanced variation between the two groups. In addition, the target population in this study was confined to patients with severe COVID-19 disease, which may have resulted in selection bias and did not provide evidence to support the use of AC treatment in patients with mild or moderate COVID-19 disease. A multicenter, retrospective study in Italy[31] and a single-center, retrospective study from Wuhan[30] also investigated the relationship between AC therapy and in-hospital mortality in COVID-19 patients. Both studies found that heparin administration was associated with lower in-hospital mortality, especially in patients with severe COVID-19 disease. Propensity score-weighting analysis was used in both these studies to control differences at baseline between the AC and non-AC users. However, an imbalance of several variates still existed between the study groups, including the disease severity classification at admission and other treatment during hospitalization which are known to be associated with the prognosis of patients with COVID-19. In our study, we used propensity score matching to create comparable study groups with balanced variates that were potential confounders associated with prognosis. However, previous research did not match the patients before grouping that would have achieved a balance between variables. We conducted the propensity score matching using covariates that included the level of oxygen therapy at admission, the most intense level of oxygen therapy during hospitalization, clinical classification at admission, hs-CRP and D-dimer levels at admission, and CURB-65 score at admission. As a consequence, the clinical classification of COVID-19 severity, ARDS classification, and oxygen therapy at admission were balanced between patients in the AC and non-AC groups. These balanced variables therefore reflected the severity of illness in COVID-19 patients at admission. One of the advantages of the current study is the strategy to use propensity score matching before grouping, which would be expected to minimize bias caused by inconsistency in the severity of illness between groups. A propensity scorematched cohort study based on hospitalized and ambulatory COVID-19 patients in New York City examined the impact on clinical outcomes of therapeutic AC administered before COVID-19 infection[32]. The covariates used for matching in that study included age, sex, race, Charlson Comorbidity Index, and obesity. However, no variates related to the severity of illness were included in the matching model. The results of the study suggested AC treatment was not protective for morbidity and mortality of COVID-19 patents.

In our retrospective cohort, we observed that therapeutic AC treatment for 7 days or longer was associated with lower in-hospital mortality of COVID-19 patients, with a multivariate Cox analysis showing that $A C$ treatment decreased the risk of in-hospital mortality (adjusted HR $=0.273$ ). We carried out a subgroup analysis in our study in order to identify the patients who might benefit from AC treatment, thereby providing insights into indications of AC treatment for clinicians. Patients were stratified according to ARDS severity classification, COVID-19 clinical classification, and D-dimer levels at hospital admission. The results showed that patients with AC treatment had greater survival benefits when they were diagnosed at admission as severe or critical clinical cases or had moderate or severe ARDS or a D-dimer level $\geq 0.5 \mu \mathrm{g} / \mathrm{mL}$, or alternatively became critical clinical cases or developed severe ARDS during hospitalization. Previous studies $[9 ; 30 ; 31]$ also carried out subgroup analyses by stratifying 
patients according to age, gender, SIC scores, and levels of D-dimer and C-reactive protein. These results provided information as to which patients with specific characteristics may benefit most from AC treatment. However, the stratification criteria used in the analyses did not include the classification of clinical severity of the target patients. It is well known that hypoxia is a core clinical manifestation and major pathophysiology change that contribute to the death of COVID-19 patients[33; 34; 35]. Both ARDS[14;23;36] and COVID-19 clinical severity classification[13;37] indicate the severity of hypoxia and are used frequently by clinicians to evaluate and triage patients and to decide major treatments (e.g., levels of oxygen therapy). In our study, we analyzed the ARDS classification and COVID-19 clinical severity classification at admission as well as during hospitalization. As a result, our findings provided more information for clinicians to determine whether to use AC treatment, not only at admission, but also when patients have progressed to severe disease levels during hospitalization.

In the analysis of safety endpoints, we observed that although the incidence of bleeding events, including hemoptysis and hematuria, were higher in the AC group compared to the non-AC group, there was no significant difference in the rate of major bleeding events, gastrointestinal bleeding, bleeding in other parts, or thrombocytopenia. In brief, the above findings suggested that the use of therapeutic AC treatment for 7 days or longer in hospitalized COVID-19 patients was associated with increased hemoptysis and hematuria but not with other bleeding events, especially major bleeding. These key observations are consistent with those reported in recent studies[28; 30].

This study had several limitations. First, it was a single-center retrospective design, and large-scale, multicenter, randomized, controlled trials are urgently needed to fully assess the efficacy of AC in patients with COVID-19. Second, as we pooled UFH, LMWH and DOACs together as AC treatments in the study, further research is required in order to determine which type of anticoagulant has the best therapeutic effect and safety profile. In addition, our study did not include patients who had receive AC treatment for $<7$ days but included patients with $A C$ treatment for $\geq 7$ days in the AC group. Whether AC treatment $<7$ days is also associated with similar outcomes and also the impact of the duration of AC treatment on the outcomes needs further investigation.

In summary, COVID-19 patients who received therapeutic AC treatment for 7 days or longer had a significantly lower in-hospital mortality with no significant increase in major bleeding events. Moreover, patients had greater survival benefit from AC treatment if they were diagnosed at admission as severe or critical cases or had moderate or severe ARDS or a D-dimer level $\geq 0.5 \mu \mathrm{g} / \mathrm{mL}$ or alternatively became critical clinical cases or developed severe ARDS during hospitalization. Our study provides new insights into the role of AC treatment in hospitalized COVID-19 patients, although further high-quality randomized, control trials are urgently needed to validate these findings.

\section{Abbreviations}

COVID-19, Coronavirus disease 2019

AC, Anticoagulation

Page 16/23 
ARDS, acute respiratory distress syndrome

SARS-CoV-2, severe acute respiratory syndrome coronavirus 2

PT, prothrombin time

LMWH, low molecular weight heparin

UFH, unfractionated heparin

PS, propensity score

hs-CRP, high-sensitivity $\mathrm{C}$ reactive protein

DOACs, direct-acting oral anticoagulants

qSOFA, quick sequential organ failure assessment

IQR, interquartile ranges

IVIG, intravenous immunoglobulin

HR, hazard ratio

SIC, sepsis-induced coagulopathy

\section{Declarations}

\section{Ethics approval and consent to participate}

This study was approved by the Medical Ethics Committee of Tongji Hospital, Tongji Medical College, Huazhong University of Science and Technology (2020-S220).

\section{Consent for publication}

Not applicable.

\section{Availability of data and materials}

Not applicable.

\section{Competing interests}

All authors have completed the ICMJE uniform disclosure form at www.icmje.org/coi_disclosure.pdf and declare funding for the submitted work as described above; no financial relationships with any 
organizations that might have an interest in the submitted work in the previous three years; no other relationships or activities that could appear to have influenced the submitted work.

\section{Funding}

This study was supported by the COVID-19 Rapid Response Research Project of Huazhong University of Science and Technology (grant 2020kfyXGYJ049, to SL).

\section{Authors' Contributions}

YB, YL, SW and SL conceptualized the paper. YB, ZH and SL had full access to all of the data in the study and take responsibility for the integrity of the data and the accuracy of the data analysis. YB, YL, ZH, YFa, GY, SY, YW, JL, YFe, LX, YZ and ZY contributed to the acquisition, analysis and interpretation of data. YB, $Y L, P Z$ and $S L$ drafted the manuscript. All authors contributed to the critical revision of the manuscript for important intellectual content and gave final approval of the version to be published. YB, SW, and JH did the statistical analysis. SL obtained the funding. The corresponding author attests that all listed authors meet authorship criteria and that no others meeting the criteria have been omitted. YB and SL supervised the study and are the guarantors.

\section{Acknowledgements}

The authors thank all the included patients and their families, physicians, nurses, dieticians, and all staff.

\section{References}

[1] T. Asselah, D. Durantel, E. Pasmant, G. Lau, and R.F. Schinazi, COVID-19: Discovery, diagnostics and drug development. J Hepatol 74 (2021) 168-184.http://dx.doi.org/10.1016/j.jhep.2020.09.031

[2] C. Huang, Y. Wang, X. Li, L. Ren, J. Zhao, Y. Hu, et al., Clinical features of patients infected with 2019 novel coronavirus in Wuhan, China. The Lancet 395 (2020) 497-506.http://dx.doi.org/10.1016/s01406736(20)30183-5

[3] M. Ackermann, S.E. Verleden, M. Kuehnel, A. Haverich, T. Welte, F. Laenger, et al., Pulmonary Vascular Endothelialitis, Thrombosis, and Angiogenesis in Covid-19. N Engl J Med 383 (2020) 120128.http://dx.doi.org/10.1056/NEJMoa2015432

[4] S.E. Fox, A. Akmatbekov, J.L. Harbert, G. Li, J. Quincy Brown, and R.S. Vander Heide, Pulmonary and cardiac pathology in African American patients with COVID-19: an autopsy series from New Orleans. The Lancet Respiratory Medicine 8 (2020) 681-686.http://dx.doi.org/10.1016/s2213-2600(20)30243-5

[5] C. Magro, J.J. Mulvey, D. Berlin, G. Nuovo, S. Salvatore, J. Harp, et al., Complement associated microvascular injury and thrombosis in the pathogenesis of severe COVID-19 infection: A report of five cases. Transl Res 220 (2020) 1-13.http://dx.doi.org/10.1016/j.trsl.2020.04.007 
[6] Y. Ding, H. Wang, H. Shen, Z. Li, J. Geng, H. Han, et al., The clinical pathology of severe acute respiratory syndrome (SARS): a report from China. J Pathol 200 (2003) 282-

9.http://dx.doi.org/10.1002/path.1440

[7] J.M. Nicholls, L.L.M. Poon, K.C. Lee, W.F. Ng, S.T. Lai, C.Y. Leung, et al., Lung pathology of fatal severe acute respiratory syndrome. The Lancet 361 (2003) 1773-1778.http://dx.doi.org/10.1016/s01406736(03)13413-7

[8] D.L. Ng, F. Al Hosani, M.K. Keating, S.I. Gerber, T.L. Jones, M.G. Metcalfe, et al., Clinicopathologic, Immunohistochemical, and Ultrastructural Findings of a Fatal Case of Middle East Respiratory Syndrome Coronavirus Infection in the United Arab Emirates, April 2014. Am J Pathol 186 (2016) 6528.http://dx.doi.org/10.1016/j.ajpath.2015.10.024

[9] N. Tang, H. Bai, X. Chen, J. Gong, D. Li, and Z. Sun, Anticoagulant treatment is associated with decreased mortality in severe coronavirus disease 2019 patients with coagulopathy. J Thromb Haemost 18 (2020) 1094-1099.http://dx.doi.org/10.1111/jth.14817

[10] L. Ayerbe, C. Risco, and S. Ayis, The association between treatment with heparin and survival in patients with Covid-19. J Thromb Thrombolysis 50 (2020) 298-301.http://dx.doi.org/10.1007/s11239020-02162-z

[11] A.S.o. hematology. COVID-19 and VTE/Anticoagulation: Frequently Asked Questions [EB/OL]. (2021). https://www.hematology.org/\%20covid-19/covid-19-and-vte-anticoagulation. [12 April, 2021]

[12] N.I.o. Health. COVID-19 Treatment Guidelines [EB/OL].

(2021). https://www.covid19treatmentguidelines.nih.gov./antithrombotic-therapy/. [12 April, 2021]

[13] N.H.C.o.t.P.s.R.o. China. Diagnosis and Treatment Protocol for COVID-19 Patients(Trial Version 8) [EB/OL].

(2020). http://www.nhc.gov.cn/yzygj/s7653p/202008/0a7bdf12bd4b46e5bd28ca7f9a7f5e5a.shtml. [19 August, 2020]

[14] A.D.T. Force, V.M. Ranieri, G.D. Rubenfeld, B.T. Thompson, N.D. Ferguson, E. Caldwell, et al., Acute respiratory distress syndrome: the Berlin Definition. JAMA 307 (2012) 252633.http://dx.doi.org/10.1001/jama.2012.5669

[15] W.H.O. (2020), Clinical management of severe acute respiratory infection when Novel coronavirus (2019-nCoV) infection is suspected: Interim Guidance. . (2020)

[16] P.C. Austin, An Introduction to Propensity Score Methods for Reducing the Effects of Confounding in Observational Studies. Multivariate Behav Res 46 (2011) 399-

424.http://dx.doi.org/10.1080/00273171.2011.568786 
[17] M.M.v.d.E. W S Lim, R Laing, W G Boersma, N Karalus, G I Town, S A Lewis, J T Macfarlane, Defining community acquired pneumonia severity on presentation to hospital: an international derivation and validation study. Thorax (2003).http://dx.doi.org/10.1136/thorax.58.5.377

[18] 0. Kuss, M. Blettner, and J. Borgermann, Propensity Score: an Alternative Method of Analyzing Treatment Effects. Dtsch Arztebl Int 113 (2016) 597-603.http://dx.doi.org/10.3238/arztebl.2016.0597

[19] S.S.a.C.K.O.B.O.T.S.O.C.O.A.O.T.S.A.S.C.O.T.I.S.O.T.A. HAEMOSTASIS, Definition of major bleeding in clinical investigations of antihemostatic medicinal products in non-surgical patients. Journal of Thrombosis and Haemostasis (2004).http://dx.doi.org/10.1111/j.1538-7836.2005.01204.x

[20] M.N. Levine, G. Raskob, and J. Hirsh, Hemorrhagic complications of long-term anticoagulant therapy. Chest 95 (1989) 26S-36S.http://dx.doi.org/10.1378/chest.95.2_supplement.26s

[21] J. Thachil, N. Tang, S. Gando, A. Falanga, M. Cattaneo, M. Levi, et al., ISTH interim guidance on recognition and management of coagulopathy in COVID-19. J Thromb Haemost 18 (2020) 10231026.http://dx.doi.org/10.1111/jth.14810

[22] I. AM Neill, RWeir,RAnderson,AChereshsky,M J Epton,RJackson, M

Schousboe,CFrampton,SHutton,STChambers,GITown, Community acquired pneumonia: aetiologyand usefulness of severitycriteriaon admission. Thorax (1996).http://dx.doi.org/10.1136/thx.51.10.1010

[23] M.D. LORRAINE B. WARE, AND MICHAEL A. MATTHAY, M.D., The acute respiratory distress syndrome. N Engl J Med (2000).http://dx.doi.org/10.1056/NEJM200005043421806

[24] E.D. Riviello, W. Kiviri, T. Twagirumugabe, A. Mueller, V.M. Banner-Goodspeed, L. Officer, et al., Hospital Incidence and Outcomes of the Acute Respiratory Distress Syndrome Using the Kigali Modification of the Berlin Definition. Am J Respir Crit Care Med 193 (2016) 52-9.http://dx.doi.org/10.1164/rccm.20150305840C

[25] M. Singer, C.S. Deutschman, C.W. Seymour, M. Shankar-Hari, D. Annane, M. Bauer, et al., The Third International Consensus Definitions for Sepsis and Septic Shock (Sepsis-3). JAMA 315 (2016) 80110.http://dx.doi.org/10.1001/jama.2016.0287

[26] S. Gando, M. Levi, and C.H. Toh, Disseminated intravascular coagulation. Nat Rev Dis Primers 2 (2016) 16037.http://dx.doi.org/10.1038/nrdp.2016.37

[27] N. Gupta, Y.Y. Zhao, and C.E. Evans, The stimulation of thrombosis by hypoxia. Thromb Res 181 (2019) 77-83.http://dx.doi.org/10.1016/j.thromres.2019.07.013

[28] I. Paranjpe, V. Fuster, A. Lala, A.J. Russak, B.S. Glicksberg, M.A. Levin, et al., Association of Treatment Dose Anticoagulation With In-Hospital Survival Among Hospitalized Patients With COVID-19. J Am Coll Cardiol 76 (2020) 122-124.http://dx.doi.org/10.1016/j.jacc.2020.05.001 
[29] S. Schulman, S. Konstantinides, Y. Hu, and L.V. Tang, Venous Thromboembolic Diseases: Diagnosis, Management and Thrombophilia Testing: Observations on NICE Guideline [NG158]. Thromb Haemost 120 (2020) 1143-1146.http://dx.doi.org/10.1055/s-0040-1712913

[30] L. Shen, L. Qiu, D. Liu, L. Wang, H. Huang, H. Ge, et al., The Association of Low Molecular Weight Heparin Use and In-hospital Mortality Among Patients Hospitalized with COVID-19. Cardiovasc Drugs Ther (2021).http://dx.doi.org/10.1007/s10557-020-07133-3

[31] A.F. Di Castelnuovo, S. Costanzo, and L. lacoviello, Heparin in COVID-19 patients is associated with reduced in-hospital mortality: the multicentre Italian CORIST Study. Thromb Haemost (2021).http://dx.doi.org/10.1055/a-1347-6070

[32] E.L. Evans, O.V. Povstyan, D. De Vecchis, F. Macrae, L. Lichtenstein, T.S. Futers, et al., Impact of anticoagulation prior to COVID-19 infection: a propensity score-matched cohort study. Blood 136 (2020) 140-144.http://dx.doi.org/10.1182/blood.2019004174

[33] V.K. Somers, T. Kara, and J. Xie, Progressive Hypoxia: A Pivotal Pathophysiologic Mechanism of COVID-19 Pneumonia. Mayo Clin Proc 95 (2020) 2339-

2342.http://dx.doi.org/10.1016/j.mayocp.2020.09.015

[34] J. Xie, N. Covassin, Z. Fan, P. Singh, W. Gao, G. Li, et al., Association Between Hypoxemia and Mortality in Patients With COVID-19. Mayo Clin Proc 95 (2020) 1138-

1147.http://dx.doi.org/10.1016/j.mayocp.2020.04.006

[35] G. Grasselli, A. Zangrillo, A. Zanella, M. Antonelli, L. Cabrini, A. Castelli, et al., Baseline Characteristics and Outcomes of 1591 Patients Infected With SARS-CoV-2 Admitted to ICUs of the Lombardy Region, Italy. JAMA 323 (2020) 1574-1581.http://dx.doi.org/10.1001/jama.2020.5394

[36] C. Ferrando, F. Suarez-Sipmann, R. Mellado-Artigas, M. Hernandez, A. Gea, E. Arruti, et al., Clinical features, ventilatory management, and outcome of ARDS caused by COVID-19 are similar to other causes of ARDS. Intensive Care Med 46 (2020) 2200-2211.http://dx.doi.org/10.1007/s00134-020-06192-2

[37] E. Nicastri, N. Petrosillo, T. Ascoli Bartoli, L. Lepore, A. Mondi, F. Palmieri, et al., National Institute for the Infectious Diseases "L. Spallanzani", IRCCS. Recommendations for COVID-19 clinical management. Infect Dis Rep 12 (2020) 8543.http://dx.doi.org/10.4081/idr.2020.8543

\section{Figures}


2469 confirmed COVID-19 patients were admitted to Tongji Hospital between Jan 25, 2020 and Mar 23, 2020

197 Excluded:

$8<18$ years

7 Pregnant

89 Re-positive cases

31 With severe comorbidities

20 Length of stay $<24$ hours

42 With insufficient medical information

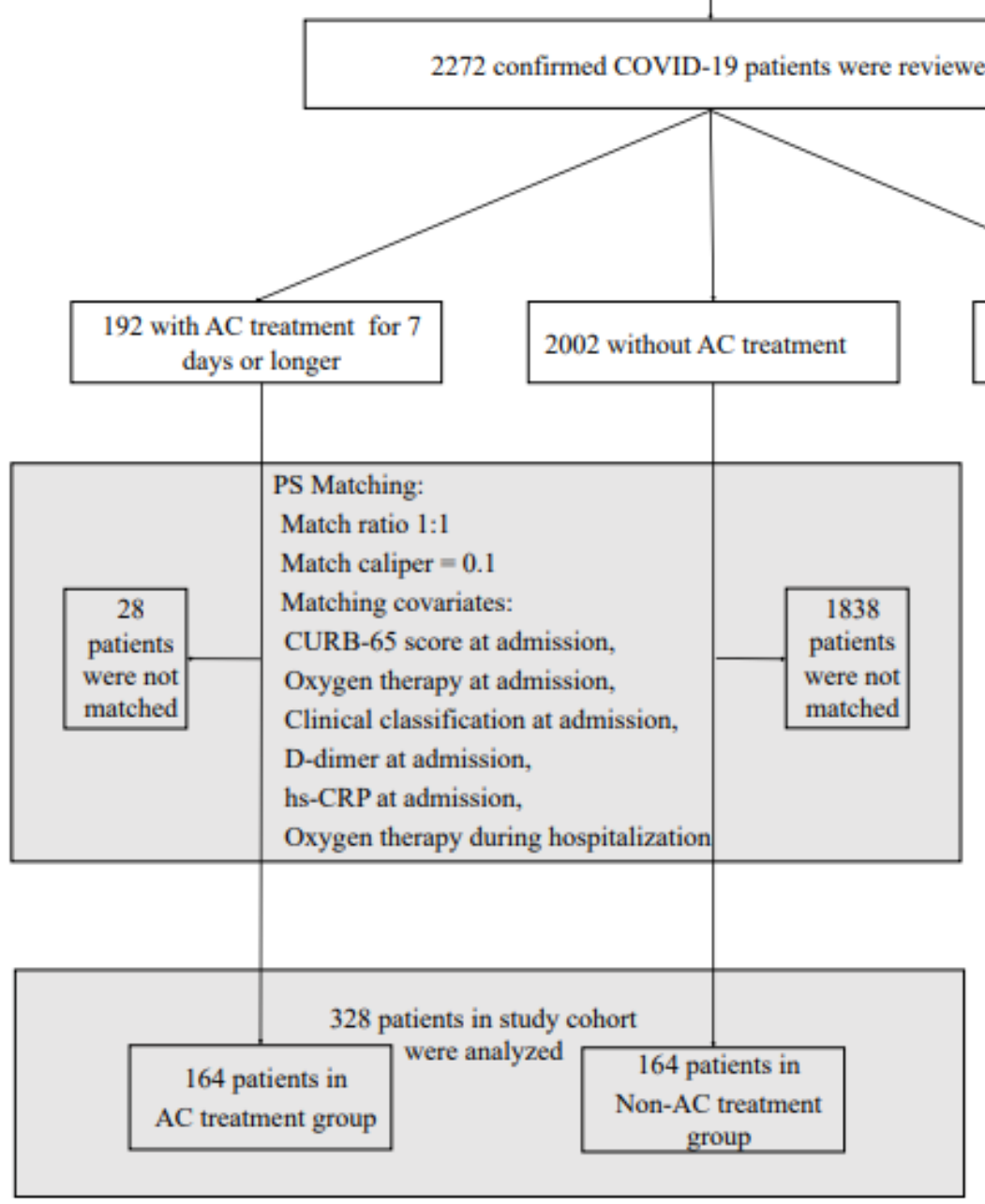

\section{Figure 1}

Flow diagram of the study Abbreviations: COVID-19, Coronavirus Disease 2019; AC, anticoagulation; PS, propensity score; hs-CRP, high sensitivity C-reactive protein 
No. at risk

$\begin{array}{lrrrrrrrrrr}\text { non-AC treatment } & 164 & 129 & 90 & 47 & 27 & 11 & 7 & 3 & 1 & 0 \\ \text { AC treatment } & 164 & 160 & 133 & 109 & 63 & 29 & 16 & 8 & 8 & 7\end{array}$

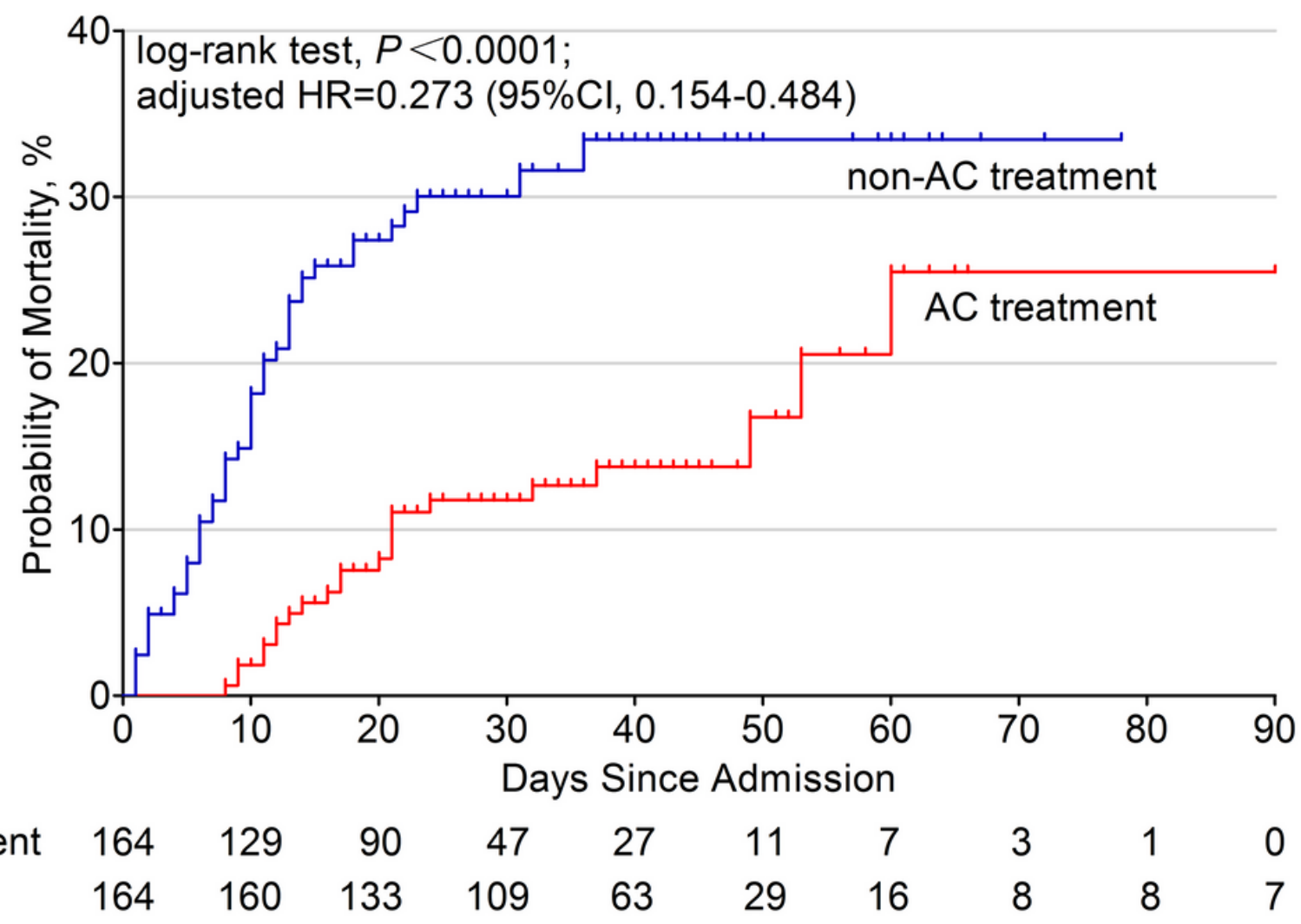

Figure 2

Cumulative probability of death in COVID-19 patients with and without AC treatment. Patients with AC treatment had a lower probability of in-hospital mortality than those without AC treatment (adjusted $\mathrm{HR}=0.273,95 \% \mathrm{Cl}: 0.154-0.484, \mathrm{P}<0.001)$. Abbreviations: HR, hazard ratio; $\mathrm{Cl}$, confidence interval; $\mathrm{AC}$, anticoagulation 